\title{
Is intestinal stasis sufficient by itself in promoting enterocolitis in a non-genetic rat model of Hirschsprung's disease?
}

\author{
Magdalini Mitroudi ${ }^{1, A-F}$, Dimitra Psalla ${ }^{2, A, B, E}$, Konstantina Kontopoulou ${ }^{3, B, E, F}$, \\ Konstantinos Theocharidis ${ }^{4, B, F}$, Dimitrios Sfoungaris ${ }^{1, A, C F}$ \\ 1 st Department of Pediatric Surgery, Aristotelion University of Thessaloniki, General Hospital G. Gennimatas, Greece \\ ${ }^{2}$ Laboratory of Pathology, School of Health Sciences, Faculty of Veterinary Medicine, Aristotelion University of Thessaloniki, Greece \\ ${ }^{3}$ Laboratory of Microbiology. G. Gennimatas General Hospital, Thessaloniki, Greece \\ ${ }^{4}$ Department of Pathology, G. Gennimatas General Hospital, Thessaloniki, Greece \\ A - research concept and design; B - collection and/or assembly of data; $\mathrm{C}$ - data analysis and interpretation; \\ $D$ - writing the article; $E$ - critical revision of the article; $F$ - final approval of the article
}

\section{Address for correspondence}

Dimitrios Sfoungaris

E-mail: dsfounga@auth.gr

Funding sources

None declared

Conflict of interest

None declared

\section{Acknowledgements}

We would like to thank Prof. Necdet Sut of Trakya University, Turkey, and Erasmus partner, for his valuable help in the statistical analysis of our results.

* These authors contributed equally to this work.

Received on July 18, 2018

Reviewed on August 26, 2018

Accepted on May 14, 2019

Cite as

Mitroudi M, Psalla D, Kontopoulou K, Theocharidis K, Sfoungaris $D$. Is intestinal stasis sufficient by itself in promoting enterocolitis in a non-genetic rat model of Hirschsprung's disease? Adv Clin Exp Med. 2019;28(10): 1429-1436. doi:10.17219/acem/109342

DOI

10.17219/acem/109342

Copyright

Copyright by Author(s)

This is an article distributed under the terms of the

Creative Commons Attribution Non-Commercial License

(http://creativecommons.org/licenses/by-nc-nd/4.0/)

\begin{abstract}
Background. Hirschsprung's disease-associated enterocolitis (HE) is a life-threatening septic complication of Hirschsprung's disease (HD), leading to bacterial translocation (BT) and sepsis. Many factors, such as intestinal stasis, HD-related inherited immune disorders and abnormal mucosal secretion have been implicated in its pathogenesis.
\end{abstract}

Objectives. To investigate the effect of intestinal stasis as an independent factor in the pathogenesis of HE intestinal lesions and its systematic effects.

Material and methods. The rectal ganglion cells of 46 Wistar rats were chemically ablated through local benzalkonium chloride (BAC) injection, in order to create a HD model (megacolon rats) that does not carry the possible genetic burden of HD. The animals were sacrificed either on the $20^{\text {th }}$ or $25^{\text {th }}$ day after ablation and were examined for histopathological changes on the wall of the small intestine, presence of bacterial translocation in body organs, body biometrics, and white blood cell count (WBC) and hemoglobin concentration. The results were compared to control animals.

Results. In the megacolon rats, severe damage on the small intestine as well as BT proportional to the extent of the intestinal damage and to the time elapsed after ablation was observed. Significant effects on the WBCs, hemoglobin concentration and biometric parameters were also observed.

Conclusions. In megacolon rats, intestinal stasis can lead by itself to a full-blown HE. The HE lesions that promote BT are present even in regions distant from the aganglionic bowel and are proportional to the time elapsed under the influence of intestinal stasis. Systematic effects such as growth retardation are also produced.

Key words: sepsis, bacterial translocation, intestinal obstruction, megacolon, ganglion cell ablation 


\section{Introduction}

Hirschsprung's disease (HD) is a congenital disease characterized by a lack of ganglion cells in a segment of the terminal bowel (aganglionosis), causing functional obstruction and distension of the proximal bowel (megacolon). ${ }^{1,2}$ In 6-26\% of HD cases, Hirschsprung's enterocolitis (HE) can develop, which is accompanied by considerable morbidity. ${ }^{2,3}$ The pathogenesis of HE appears complex and is not completely understood. ${ }^{4}$ It presents with both gastrointestinal (GI) and generalized symptoms ranging from fever and diarrhea to bloodstained stools and septic shock, while in chronic cases it affects somatic growth. ${ }^{5-7}$

Initially, gradual intestinal obstruction, bacterial overgrowth and bacterial translocation (BT) were proposed as causative mechanisms. ${ }^{8}$ However this has been repeatedly questioned, because $\mathrm{HE}$ can occur even after surgical correction of HD, or after decompressing colostomy. ${ }^{9}$ This led to the investigation of additional possible $\mathrm{HE}$ causative factors, such as deficiency of the immune system and compromise of local intestinal mucus production. ${ }^{10-12}$ These factors could be inherited through complex HD genetics. ${ }^{4}$

The goal of this study was to investigate whether aganglionosis-induced intestinal stasis can by itself cause HE. In order to eliminate the interference of other possible HD-related factors, we used an animal model without HD genetic burden, in which aganglionosis was created through local chemical ablation of ganglion cells. We investigated the effects of intestinal stasis by studying the degree of intestinal damage, the degree of BT and the effects on somatic growth. This information could lead to a better understanding of the pathophysiology of HE and to a more targeted and timely management of the patients.

\section{Material and methods}

\section{Animals}

A total of 62 Wistar rats aged 28 days were used. All the rats were obtained from the same breeding center. They were housed in macrolon cages, 1 rat per cage, at $20-22^{\circ} \mathrm{C}$ room temperature. The rats were fed with standard rat chow diet.

Five of our animals were used in a pilot study to establish the appropriate time for sacrifice, in order to study the animals at 2 different stages of disease, but without death ensuing. These rats were ablated as described below and monitored at least once a day for symptoms of decreased activity, poor feeding and abdominal distension; stool production, weight and height (nose to rump length) were also measured. We observed that at least 2 symptoms were present by the $18^{\text {th }}$ day after ganglion cell ablation. Two rats, randomly selected, were sacrificed on the $20^{\text {th }}$ day posttreatment and 3 on the $25^{\text {th }}$ day. These animals were assigned respectively to the M20 and M25 groups of animals, according to the investigative protocol, as described below.

\section{Experimental design}

The animals were randomly assigned to 4 groups as follows: group $1(\mathrm{~N})(\mathrm{n}=8)$ - animals with no treatment; group $2(\mathrm{~S})(\mathrm{n}=8)$ : sham animals, injected with saline in the submucosa of the colon; group 3 (M20) ( $n=23)$ : megacolon animals, treated for chemical ablation, as described below, sacrificed on the $20^{\text {th }}$ day post-treatment; group 4 (M25) ( $\mathrm{n}=23)$ : megacolon animals, treated for chemical ablation, as described below, sacrificed on the $25^{\text {th }}$ day post-treatment. We refer to group $1(\mathrm{~N})$ and group $2(\mathrm{~S})$ collectively as "ganglionic" animals. We refer to group 3 (M20) and group 4 (M25) collectively as "megacolon" animals.

The animal protocol was approved by the State Institutional Animal Care and Use Committee and by the Ethics Committee of Animal Research, Aristotelion University of Tessaloniki.

At 28 days of age, nonfasted ganglionic littermates (groups $\mathrm{N}$ and $\mathrm{S}$ ) were anesthetized by intramuscular injection of $30 \mathrm{mg} / \mathrm{kg}$ ketamine hydrochloride. Body weight and body height were measured. Blood was collected from the lateral tail vein at the start of the study and from the aorta at the time of sacrifice.

The animals of groups M20 and M25 were anesthetized, positioned supine and were injected at 8 points of the rectum circumference between $0.2 \mathrm{~cm}$ to $1 \mathrm{~cm}$ above the anal verge with $50 \mu \mathrm{L}$ of $0.1 \%$ benzalkonium chloride (BAC) solution in deionized water. A 22-gauge needle attached to a $50-\mu \mathrm{L}$ micro syringe was used. The injection site was marked with India ink 1:100 (vol/vol). Group S animals were subjected to the same procedure using $0.9 \% \mathrm{NaCl}$ solution instead of BAC. There was no intervention in group N.

On the day of sacrifice, the animals were anesthetized. Their abdominal fur was shaved and the skin was cleaned and disinfected with iodine solution. The abdomen was opened through a $4 \mathrm{~cm}$ midline incision. Blood was collected from the abdominal aorta and the internal organs were collected under aseptic conditions for bacteriological investigation. The rats were then killed by infusing potassium chloride $(\mathrm{KCl})$ in the heart.

\section{Organ sampling for bacterial translocation assessment}

For BT assessment, the mesenteric lymph nodes (MLN), spleen, liver, kidneys, and lungs were collected separately, weighed and homogenized in $9 \mathrm{~mL}$ sterile pre-reduced 1:4 Ringer's solution, seeded in culture media and incubated. Each type of colony was submitted to Gram staining, catalase assay and anaerobic assay.

\section{Blood sampling}

White blood cells were evaluated according to standard laboratory methods. For hematological analyses, $1 \mathrm{~mL}$ of blood was collected in an anticoagulant 
(ethylenediaminetetraacetic acid (EDTA)) tube. A Coulter T890 ${ }^{\circledR}$ device (Beckman Coulter, Brea, USA) was used for white blood cell count (WBC).

\section{Histological preparation}

The entire intestine (including the anus) was excised, washed gently with cold phosphate-buffered saline (PBS) and fixed with $10 \%$ neutral-buffered formalin at room temperature for 48-72 h (Fig. 1). Specimens $1 \mathrm{~cm}$ long were sampled from the terminal ileum $(5 \mathrm{~cm}$ proximally to the ileocolic valve) and cut into segments. All specimens were immersed in alcohol and xylol, and then embedded in paraffin. Examination was performed on 4-6 $\mu \mathrm{m}$ hematoxylin and eosin (H\&E)-stained sections. Four sections from each rat were examined. Light microscopic studies were reviewed in a blinded manner. Tissue damage, severity and the depth of inflammation were evaluated, according to criteria listed below.

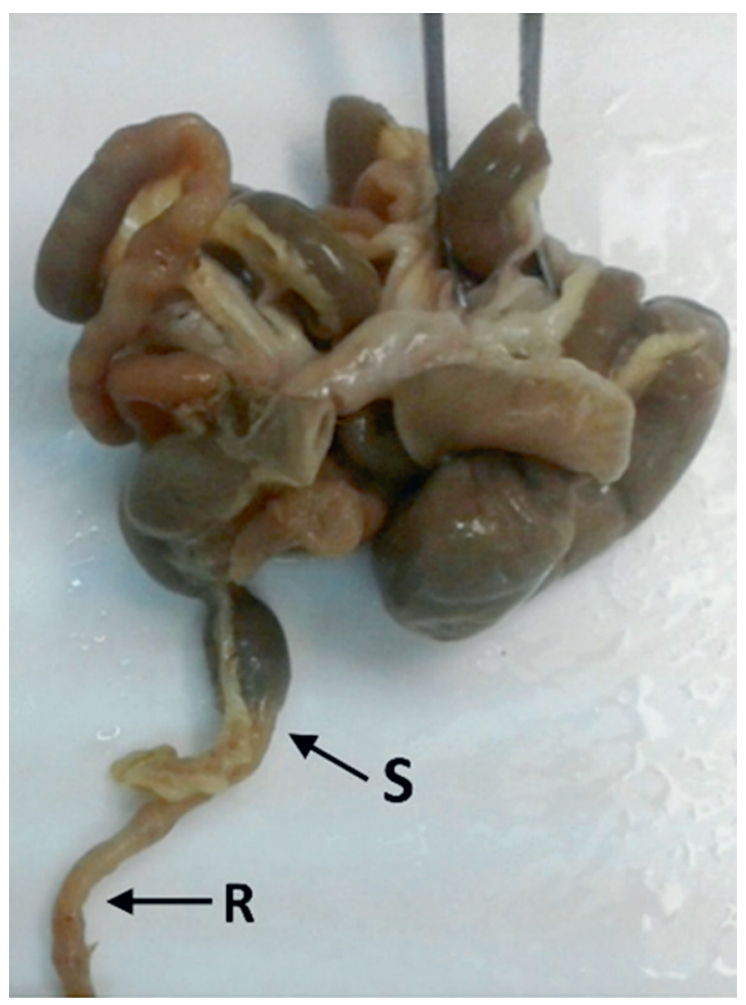

Fig. 1. Intestine of megacolon rat showing the reduced diameter of the rectum (R) and sigmoid (S)

\section{Histological grading system}

Mucosal lesion damage was assessed and graded according to the "tissue damage degree" criteria proposed by Chiu et al. ${ }^{13}$ (Table 1 ). The severity and depth of inflammation were scored separately according to the histological classification system described by Cheng et al. ${ }^{14}$ (Table 1). The highest score obtained in each parameter in each animal was recorded. Their sum represented
Table 1. Histological findings, total damage and enterocolitis grading system

\begin{tabular}{|c|c|}
\hline Histological findings & GRADE \\
\hline \multicolumn{2}{|l|}{ Tissue damage } \\
\hline Normal structure of villi & 0 \\
\hline $\begin{array}{l}\text { Development of small subepithelial space at the villous } \\
\text { apex }\end{array}$ & 1 \\
\hline $\begin{array}{l}\text { Enlarged subepithelial space but without change } \\
\text { in villous length and width }\end{array}$ & 2 \\
\hline Few shortened villi and presence of cells in the lumen & 3 \\
\hline $\begin{array}{l}\text { The majority of villi are shortened and widened with } \\
\text { crypt hyperplasia and cells in the lumen }\end{array}$ & 4 \\
\hline $\begin{array}{l}\text { Blunting of all villi with elongated crypts and increased } \\
\text { number of cells in the lumen }\end{array}$ & 5 \\
\hline \multicolumn{2}{|l|}{ Severity of inflammation } \\
\hline No inflammation, rare neutrophil & 0 \\
\hline Mild inflammatory infiltrates, no necrosis & 1 \\
\hline $\begin{array}{l}\text { Moderate to marked inflammatory infiltrates and } \\
\text { mucosal necrosis }\end{array}$ & 2 \\
\hline Transmural necrosis & 3 \\
\hline \multicolumn{2}{|l|}{ Depth of inflammation } \\
\hline None & 0 \\
\hline Mucosa & 1 \\
\hline Submucosa & 2 \\
\hline Muscularispropria & 3 \\
\hline Subserosa and serosa & 4 \\
\hline $\begin{array}{l}\text { Total enterocolitis score } \\
\text { Severity of inflammation grade }+ \text { depth of inflammation } \\
\text { grade }\end{array}$ & range: $0-7$ \\
\hline $\begin{array}{l}\text { Total damage score } \\
\text { Total enterocolitis score + tissue damage grade }\end{array}$ & range: $0-12$ \\
\hline
\end{tabular}

the "total enterocolitis score". ${ }^{14}$ To take under consideration all the above parameters, we used a combined score - the "total damage score", calculated by adding the "total enterocolitis score" and "tissue damage grade" (Table 1).

\section{Statistical analysis}

Before the beginning of the study, a sample size calculation was performed with $80 \%$ power and an error set at 0.05 (two-sided). The Kruskal-Wallis test was used for comparison of the parameters due to the non-normal distribution. Then, the Mann-Whitney U test was used for pairwise comparisons of the groups when a significant difference was obtained. The data was presented as means and standard deviation (SD), and $\mathrm{p}<0.05$ was considered statistically significant.

\section{Results}

All rats survived during the experimental period. The histological examination of the BAC-treated rectums confirmed the ablation of intestinal ganglia in all of these 
Table 2. Comparison of histological findings, hematological findings, weight (Weight_e) and height (Height e) between the groups at the time of sacrifice. Means \pm SD are reported. P-value $<0.05$ indicates groups are significantly different

\begin{tabular}{|c|c|c|c|c|c|}
\hline \multirow[b]{2}{*}{ Variable } & \multicolumn{4}{|c|}{ Groups } & \multirow[b]{2}{*}{$\mathrm{p}$-value } \\
\hline & $\begin{array}{c}\text { normal }(n=8) \\
\text { mean } \pm S D\end{array}$ & $\begin{array}{c}\text { sham }(n=8) \\
\text { mean } \pm S D\end{array}$ & $\begin{array}{c}M 20(n=23) \\
\text { mean } \pm S D\end{array}$ & $\begin{array}{c}\mathrm{M} 25(\mathrm{n}=23) \\
\text { mean } \pm \mathrm{SD}\end{array}$ & \\
\hline Tissue damage & $0 \pm 0$ & $0 \pm 0$ & $3.1 \pm 1.3$ & $4.7 \pm 0.5$ & $<0.001$ \\
\hline Severity of inflammation & $0 \pm 0$ & $0 \pm 0$ & $1.6 \pm 0.9$ & $2.5 \pm 0.5$ & $<0.001$ \\
\hline Depth of inflammation & $0 \pm 0$ & $0 \pm 0$ & $1.1 \pm 1.1$ & $3.2 \pm 0.9$ & $<0.001$ \\
\hline Total damage score & $0 \pm 0$ & $0 \pm 0$ & $6 \pm 3$ & $10.4 \pm 1.7$ & $<0.001$ \\
\hline Height_e [cm] & $27.63 \pm 1.99$ & $27.00 \pm 2.56$ & $22.35 \pm 1.22$ & $22.78 \pm 0.99$ & $<0.001$ \\
\hline Weight_e [g] & $243.00 \pm 1.77$ & $242.88 \pm 1.88$ & $231.48 \pm 3.14$ & $230.78 \pm 2.02$ & $<0.001$ \\
\hline WBC_e $\left[10^{3} / \mu \mathrm{L}\right]$ & $7.15 \pm 1,628.32$ & $7,362.50 \pm 1,281.67$ & $11,947.82 \pm 4,775.11$ & $13,234.34 \pm 8,554.30$ & $<0.017$ \\
\hline NEUe $\left[10^{3} / \mu \mathrm{L}\right]$ & $3,383.38 \pm 722.85$ & $3,913.38 \pm 973.06$ & $10,061.70 \pm 4,311.34$ & $11,588.35 \pm 7,840.43$ & $<0.001$ \\
\hline Hb_e [g/dL] & $12.53 \pm 1.01$ & $12.37 \pm 1.06$ & $10.53 \pm 1.54$ & $11.29 \pm 1.30$ & 0.001 \\
\hline
\end{tabular}

SD - standard deviation; Weight_e - body weight at the time of sacrifice; Height_e - body height at the time of sacrifice; WBC - white blood cells; NEU - neutrophils; $\mathrm{Hb}$ - hemoglobin.

animals. Non BAC-treated animals (Normal and Sham groups) did not produce histological signs of ganglion ablation, as expected.

\section{Biometric parameters}

Between the $17^{\text {th }}$ and the $20^{\text {th }}$ day, the megacolon animals began eating less food and produced less fecal material. By the $25^{\text {th }}$ day, all megacolon animals showed decreased activity, poor feeding, less stool production, and abdominal distension. All rats continued to gain weight and height. However, the body weight at the time of sacrifice in both M20 group (M20 Weight_e: $231.48 \pm 3.14 \mathrm{~g}$ ) and M25 group (M25 Weight_e: $230.78 \pm 2.02 \mathrm{~g}$ ) remained significantly lower, compared to the respective parameters in the ganglionic groups $(\mathrm{p}<0.001)$. The body height at the time of sacrifice in the M20 group (M20 Height_e: $22.35 \pm 1.22 \mathrm{~cm}$ ) and M25 group (M25 Height_e: $22.78 \pm 0.99 \mathrm{~cm})$ remained significantly lower compared to the rats of the ganglionic groups $(\mathrm{p}<0.001)$ (Table 2 , Fig. 2,3). Differences in weight and height between the M20 and M25 groups were not statistically significant.

\section{Histological examination of the terminal ileum}

No injury or inflammation was observed in the $\mathrm{N}$ and $S$ groups (Fig. 4), which showed normal mucosa and glands. However, in the M20 and M25 groups, the intestinal structure was damaged (Fig. 5,6). Epithelial ulceration and infiltration with a mixed leukocyte population were observed. Denuded villi were discovered, accompanied by expansion of the subepithelial space with moderate or massive epithelial elevation. The total damage score in the M25 group was significantly higher than in the M20 group (total damage - M20: $6 \pm 3$ vs M25: $10.4 \pm 1.7)(\mathrm{p}<0.001)$ (Table 2$)$.

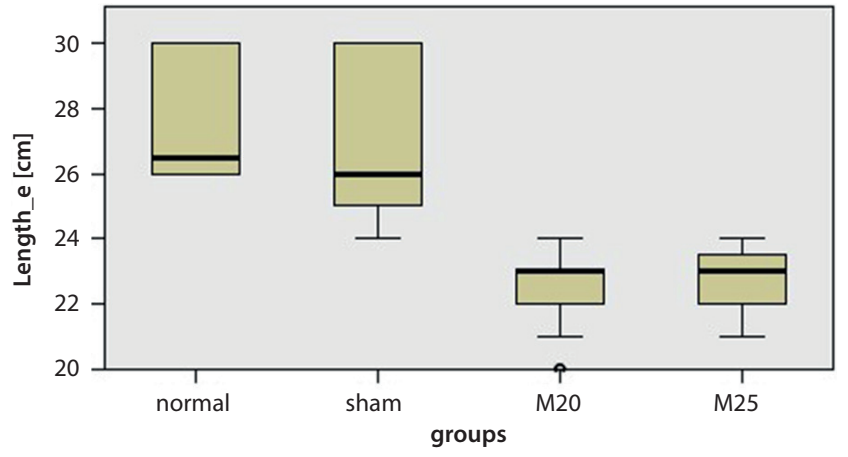

Fig. 2. Nose to rump length at sacrifice (Length_e) [cm] in N, S, M20, and M25 groups at the time of sacrifice (means \pm SD)

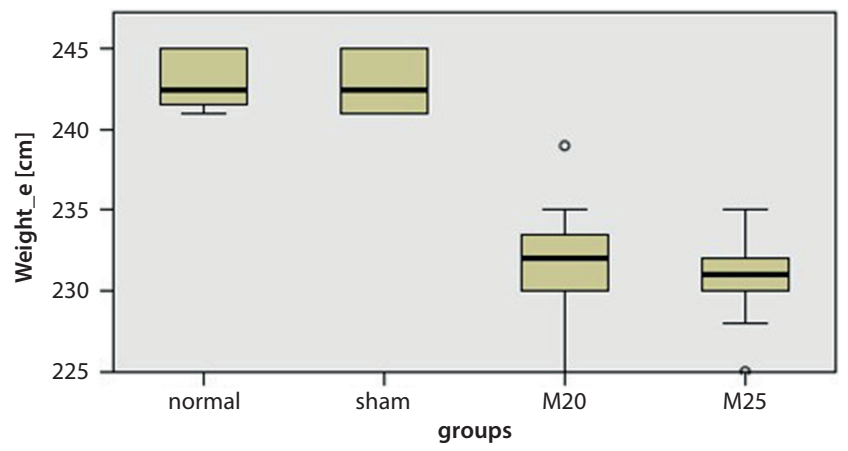

Fig. 3. Weight (Weight_e) [g] in N, S, M20, and M25 groups at the time of sacrifice (means \pm SD)

\section{Bacterial translocation}

All rats were tested for the presence of bacteria in their MLN, liver, spleen, kidneys, and lungs. No bacteria were cultured from these organs among the control and sham rats. Escherichia coli, Enterococcus spp., Bacillus, Proteus mirabilis, and Clostridium spp. were isolated only among the megacolon groups. Escherichia coli translocated at a higher incidence compared to other bacteria. 


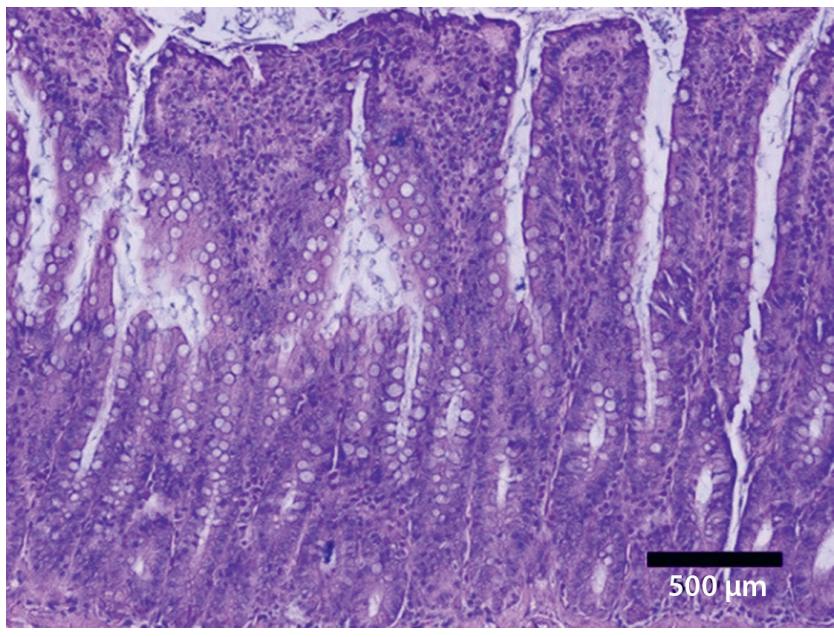

Fig. 4. Sham animal. Histological section showing intact epithelium. The number of lymphocytes and plasma cells in the lamina propria are within normal limits. H\&E staining, bar: $500 \mu \mathrm{m}$

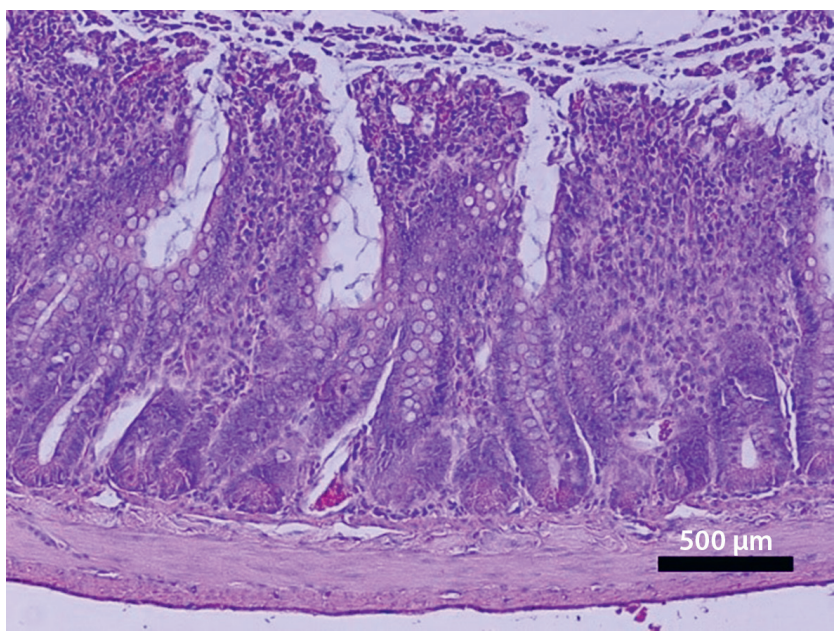

Fig. 5. Megacolon animal, M20 group. Histological section demonstrating ulceration of the epithelium and moderate infiltration by a mixed leukocyte population. H\&E staining, bar: $500 \mu \mathrm{m}$

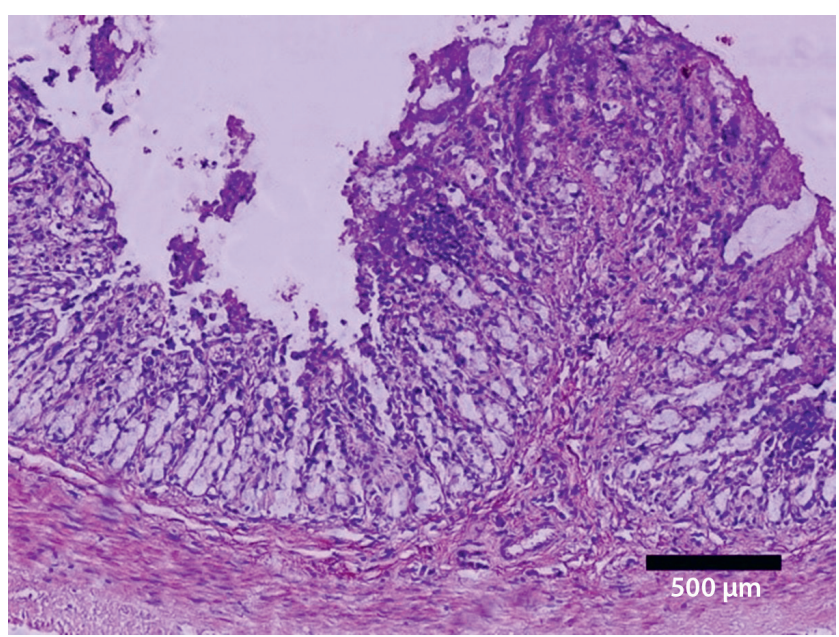

Fig. 6. Megacolon animal, M25 group. Histological section demonstrating severe destruction/necrosis of the epithelium extending to the lamina propria and infiltration by leukocytes. H\&E staining, bar: $500 \mu \mathrm{m}$
Escherichia coli was observed in $73.9 \%$ of the rats in the M20 group and, more specifically, in $65.21 \%$ of the mesenteric lymph nodes, $34.78 \%$ of the spleens, $30.43 \%$ of the livers, $26.08 \%$ of the kidneys, and $4.34 \%$ of the lungs. All M25 rats showed viable $E$. coli bacteria in extra-intestinal sites: in $100 \%$ of the MLN, $78.26 \%$ of the spleens, $91.3 \%$ of the livers, $69.56 \%$ of the kidneys, and $60.86 \%$ of the lungs.

\section{Hematological findings}

The megacolon rats showed a significant increase in the number of WBC and neutrophils (NEU) compared to the ganglionic rats (Table 2, Fig. 7,8). Among the M20 and M25 groups, WBC count was significantly different (M20 WBC_e: $11,947.82 \pm 4,775.11 / \mu \mathrm{L}$ vs M25

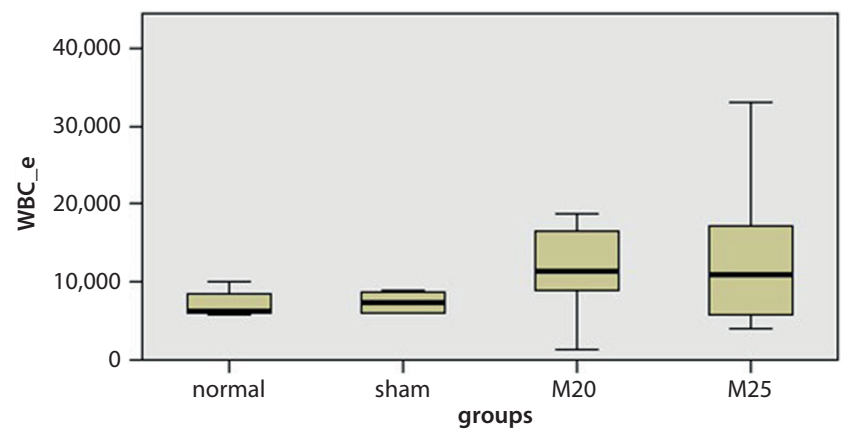

Fig. 7. White blood cells (WBC) in N, S, M20, and M25 group at the end of the experiment (means \pm SD)

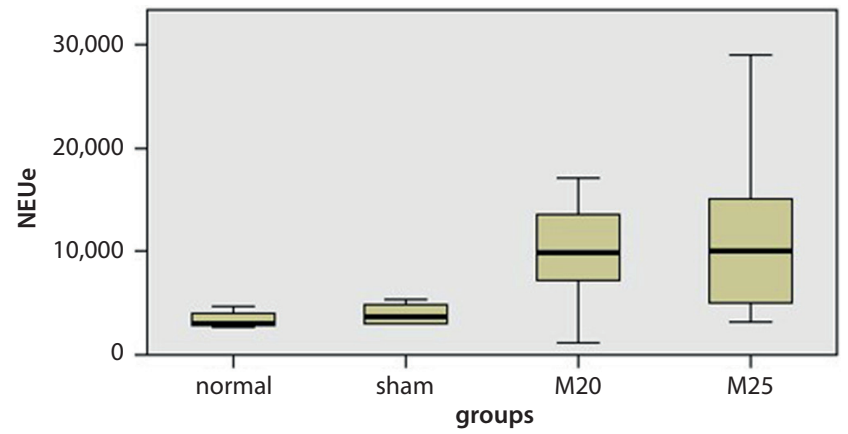

Fig. 8. Number of neutrophils (NEU) in N, S, M20, and M25 groups at the end of the experiment (means \pm SD)

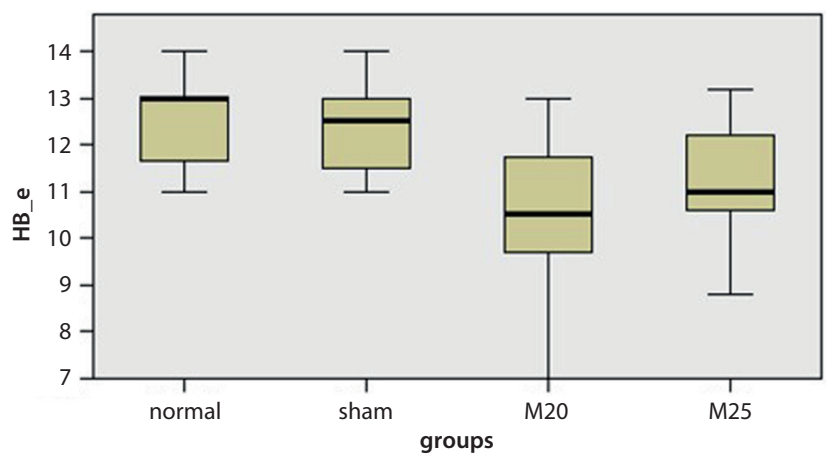

Fig. 9. Hemoglobin concentration levels $(\mathrm{Hb})[\mathrm{gr} / \mathrm{dL}]$ in $\mathrm{N}, \mathrm{S}, \mathrm{M} 2 \mathrm{O}$, and $\mathrm{M} 25$ groups at the end of the experiment (means \pm SD) 
WBC_e: $13,234.34 \pm 8,554.3 / \mu \mathrm{L})$ and NEU (M20 NEUe: $10061.70 \pm 4,311.34 / \mu L v s$ M25NEUe: $11,588.35 \pm 7,840.43 / \mu \mathrm{L})$.

There was a significant reduction in hemoglobin concentration between the ganglionic and the megacolon groups (normal Hb_e: $12.53 \pm 1.01$ gr/dL vs M20 Hb_e: $10.53 \pm 1.54$ gr/dL vs M25 Hb_e: $11.29 \pm 1.3 \mathrm{gr} / \mathrm{dL}$ ) (Table 2, Fig. 9). There was no significant difference between the M20 and M25 groups $(\mathrm{p}=0.117)$.

\section{Discussion}

Hirschsprung's enterocolitis is characterized by severe inflammation of the intestinal wall, intestinal distention and sepsis that can lead to death. ${ }^{1}$ Many factors have been studied to explain the etiology and development of HE as well as its consequences.

Historically, the first proposed etiologic mechanism for $\mathrm{HE}$ was fecal stasis proximally to the aganglionic segment causing intestinal distention, which in turn increased intestinal permeability and promoted bacterial invasion and translocation. However, this mechanism does not explain neither the occurrence of HE in patients with HD without distended bowel (such as those after decompressive colostomy, or those with surgically corrected HD) nor the presence of HE lesions in the aganglionic bowel segment. ${ }^{11,15}$

This paradox has led researchers to investigate the effects of many other factors of the development of HE, acting independently or in concert, such as microbiome alteration and impaired mucosal barrier function. These can be caused by variations in the components and amount of mucus, immunological deficiency, either local or systemic, with deficient white cell function, mucosal immunity defects, increased prostaglandin E1 activity, impaired motility associated with protein sensitization, and sucrase-isomaltase deficiency. ${ }^{11,12,16-20}$ These conditions could be the expression of the complex genetics of HD. ${ }^{21}$ Increased HE risk in patients with Down syndrome, ${ }^{22,23}$ cartilage-hair hypoplasia, ${ }^{24}$ family history of HD, and female sex point to a possible genetic contribution to the etiology of $\mathrm{HE} .{ }^{25}$ The presence of major associated congenital anomalies involving either the cardiac, GI, genitourinary, or central nervous system is also correlated with a more severe course of HE. ${ }^{21}$

Despite the fact that an ever-growing number of HDassociated mutations have been shown to be associated with a more severe morbidity, especially in the Ret-GDNF and $E T-3-E D N R B$ genes, no precise genetic abnormality has been shown to cause HE. ${ }^{26,27}$

Several studies report that HE seems to be more common in patients with a longer aganglionic segment, but it is not clear whether this tendency is related to the underlying long-segment HD genetic predisposition. ${ }^{28}$

Other risk factors not connected with genetic predisposition have also been implicated in the development of $\mathrm{HE}$. Among these is delay in HD diagnosis, the type of surgical operation, and the development of postoperative complications such as strictures and obstruction. ${ }^{9,29}$ These factors could act by creating reservoirs for stagnant intestinal contents that favor chronic inflammation, ulceration and impaired motility.

In order to exclude the confounding factors present in genetic HD animal models, we used a chemical model of HD instead of a genetic model and we investigated the effects of intestinal stasis on the intestinal wall, which is not adjacent to the aganglionic segment and not directly affected by the alterations in the mucosa caused by induced aganglionosis. We also examined the presence and extent of BT under the absence of HD-related genetic factors.

In order to ablate the ganglion cells and create HD conditions, benzalkonium chloride (BAC) was injected into the anorectal wall of normal rats. It is a cationic surfactant agent that adheres to the cell membrane, causing irreversible depolarization, injuring the cell membrane and producing severe cell damage and cell death. ${ }^{30}$ Its use for this purpose was first described by Sato et al. in $1978 .^{30}$ Researchers have practiced BAC injections either directly per annum or during laparotomy. ${ }^{30-32}$

We used the per annum endorectal injection model, because it causes less mortality. ${ }^{32}$ It has been observed that ganglion cell ablation occurs around the $14^{\text {th }}$ day after BAC treatment, as evidenced by histology ${ }^{32}$ and clinical symptomatology.

To our knowledge, experimental studies on $\mathrm{HE}^{33-35}$ have only been performed on genetic HD models. Fujimoto et al., using the piebald-lethal (PL) model, ${ }^{34,35}$ reported significant histological evidence of enterocolitis in the ganglionic portion of the colon, accompanied by acute splenitis, but no bacterial cultures were performed.

On the contrary, Caniano et al., using the same model, ${ }^{10}$ found no evidence of enterocolitis in the colon, whereas BT was present in only $10 \%$ of mice sacrificed between 12 and 70 days of age. However, BT was present in $38 \%$ of spontaneously dying mice. In their opinion, these findings suggest that other mechanisms, in addition to local colonic inflammation, contribute to the development of HE. By studying IgG, IgM and IgA levels, they concluded that a reduction in immunologic integrity was the reason for sepsis. However, their search for BT and HE lesions was conducted only on the spleen and the colon, respectively. Although the genetic model used in the 2 abovementioned experiments was the same, the lack of predetermined criteria for enterocolitis and differences in animal care and housing conditions may have contributed to different results. Other investigators have performed detailed studies on the $\mathrm{HE}$ lesions and patterned classifications regarding the grade of inflammation. ${ }^{14}$

Our findings suggest that HE lesions can develop after fecal stasis, regardless of genetic or other local contributing factors. These can appear even in the small bowel, away from the obstruction site. These lesions could act as gateways for BT through the lymphatics and mucosal blood vessels towards the portal and systematic circulation. ${ }^{36}$ 
Fecal stasis can also develop after scarring of severe intestinal lesions or adhesions following the operation for HD stasis and may trigger new HE lesions. ${ }^{37}$ These possible events may explain HE occurring in patients with successful HD surgery or who carry a stoma. ${ }^{9}$

We investigated many extra-intestinal sites for BT and we found a strong positive correlation between total intestinal damage score and cultured bacteria on MLN, liver, spleen, pancreas, kidney, and lung tissues in our megacolon rats, suggesting a causal relation between HE lesions and sepsis. Bacterial translocation was observed in the MLN in a considerably larger number of cases than in the spleen or other organs. Escherichia coli translocated to a higher frequency compared to other bacteria and this could be the consequence of its abundant colonization in the bowel. During the 5-day interval between the M20 and M25 groups, there was a significant progression of the tissue damage and increase of inflammation markers, as well as increased frequency and spread of BT, even to the lungs. This coincided with a deterioration of body homeostasis, as shown by WBC counts and biometric parameters.

The increase in WBC and NEU counts observed in the megacolon groups is consistent with the presence of intestinal inflammation and BT (Table 2) (Fig. 3,4). However, the increase of WBC count between the M20 and M25 groups, while statistically significant, was not proportional to the escalation of the intestinal inflammation and BT, which may be due to the variable response of individual mice to the extreme septic burden. In some of these, overwhelming sepsis suppresses the bone marrow and reduces WBC production ${ }^{38}$ while in others the immune system is still able to react more effectively. It is likely that for this reason, the WBC values obtained in the M25 group are more widespread than in other groups (Fig. 7, Table 2).

In the present study, all of the megacolon rats experienced reduced food intake and decreased excretion of feces by the $18^{\text {th }}$ day after endorectal injections. This was reflected in their biometric growth indices in the M20 and M25 groups (Table 2). This is in line with the findings of other researchers, that anorexia, reduced caloric intake and increased catabolism are prominent during sepsis. ${ }^{39}$

The present study suggests that the intestinal stasis itself may be the causative agent that can affect the intestine, even at a distance, causing $\mathrm{HE}$ and leading to bacterial translocation, systemic inflammation and sepsis. The development of HE lesions is gradual, and so is the intensity and extent of BT. Sepsis could affect the growth and development of the animal's body both through decreased food intake and increased catabolism.

\section{Conclusions}

Our study shows that HE can develop as a consequence of intestinal stasis alone.
Functional intestinal obstruction results in inflammation of the intestinal wall and BT to regional lymph nodes and distally located organs. Escherichia coli translocate to extra-intestinal sites at a higher frequency compared to other bacteria. The severity of the lesions of HE and the extent of $\mathrm{BT}$ is proportional to the time elapsed since the onset of intestinal stasis. Systematic effects, such as an increase in WBC and NEU counts, are also prominent. The decrease in hemoglobin concentration and restraint of somatic growth may result from inflammatory stress, sepsis and reduced food intake.

\section{ORCID iDs}

Magdalini Mitroudi (D https://orcid.org/0000-0002-5181-8887 Dimitra Psalla (D) https://orcid.org/0000-0002-1539-4124 Konstantina Kontopoulou (D) https://orcid.org/0000-0003-0121-8688 Konstantinos Theocharidis (D) https://orcid.org/0000-0001-7213-6925 Dimitrios Sfoungaris (D) https://orcid.org/0000-0001-5567-5118

\section{References}

1. Hirschsprung $\mathrm{H}$. Stuhtragheit Neugeborener infolge Dilatationen und Hypertrophie des Colons. Jahrbuch Kinderheikunde. 1887;27:1.

2. Pastor AC, Osman F, Teitelbaum DH, Caty MG, Langer JC. Development of a standardized definition for Hirschsprung's-associated enterocolitis: A Delphi analysis. J Pediatr Surg. 2009;44(1):251-256.

3. Elhalaby EA, Coran AG, Blane CE, HirschI RB, Teitelbaum DH. Enterocolitis associated with Hirschsprung's disease: A clinical-radiological characterization based on 168 patients. J Pediatr Surg. 1995;30(1):76-83.

4. Pini Prato A, Rossi V, Avanzini S, Mattioli G, Disma N, Jasonni V. Hirschsprung's disease: What about mortality? Pediatr Surg Int. 2011;27(5): 473-478.

5. Carneiro PMR, Brereton RJ, Drake DP, Kiely EM, Spitz L, Turnock R. Enterocolitis in Hirschsprung's disease. Pediatr Surg Int. 1992;7(5): 356-360.

6. Ouladsaiad M. How to manage a late diagnosed Hirschsprung's disease. Afr J Paediatr Surg. 2016;13(2):82-87.

7. Langer JC. Hirschsprung disease. Curr Opin Pediatr. 2013;25(3):368-374.

8. Coran AG, Teitelbaum DH. Recent advances in the management of Hirschsprung's disease. Am J Surg. 2000;180(5):382-387.

9. Hackam DJ, Filler RM, Pearl RH. Enterocolitis after the surgical treatment of Hirschsprung's disease: Risk factors and financial impact. J Pediatr Surg. 1998;33(6):830-833.

10. Caniano DA, Teitelbaum DH, Qualman SJ, Shannon BT. The piebaldlethal murine strain: Investigation of the cause of early death. J Pediatr Surg. 1989;24(9):906-910.

11. Jiao CL, Chen XY, Feng JX. Novel insights into the pathogenesis of Hirschsprung's-associated enterocolitis. Chin Med J (Engl). 2016; 129(12):1491.

12. Murphy F, Prem P. New insights into the pathogenesis of Hirschsprung's associated enterocolitis. Pediatr Surg Int. 2005;21(10): 773-779.

13. Chiu CJ, McArdle AH, Brown R, Scott HJ, Gurd FN. Intestinal mucosal lesion in low-flow states. I. A morphological, hemodynamic, and metabolic reappraisal. Arch Surg. 1970;101(4):478-483.

14. Cheng Z, Dhall D, Zhao L, et al. Murine model of Hirschsprung-associated enterocolitis. I. Phenotypic characterization with development of a histopathologic grading system. J Pediatr Surg. 2010;45(3):475-482.

15. Rossi V, Avanzini S, Mosconi M, et al. Hirschsprung-associated enterocolitis. J Gastroint Dig Syst. 2014;4:170.

16. Gosain A, Brinkman AS. Hirschsprung's-associated enterocolitis. Curr Opin Pediatr. 2015;27(3):364-369.

17. Wilson-Storey D, Scobie WG. Impaired gastrointestinal mucosal defense in Hirschsprung's disease: A clue to the pathogenesis of enterocolitis? J Pediatr Surg. 1989;24(5):462-464.

18. Fujimoto T, Miyano T. Abnormal expression of the blood group antigen (BGA) in colon of Hirschsprung's disease. Pediatr Surg Int. 1994; 9(4):242-247. 
19. Wilson-Storey D, Scobie WG, Raeburn JA. Defective white blood cell function in Hirschsprung's disease: A possible predisposing factor to enterocolitis. J R Coll Surg Edinb. 1988;33(4):185-188.

20. Teitelbaum DH, Qualman SJ, Caniano DA. Hirschsprung's disease: Identification of risk factors for enterocolitis. Ann Surg. 1988;207(3): 240-244.

21. Puri P, Shinkai T. Pathogenesis of Hirschsprung's disease and its variants: Recent progress. Semin Pediatr Surg. 2004;13(1):18-24.

22. Menezes M, Puri P. Long-term clinical outcome in patients with Hirschsprung's disease and associated Down's syndrome. J Pediatr Surg. 2005;40(5):810-812.

23. Morabito A, Lall A, Gull S, Mohee A, Bianchi A. The impact of Down's syndrome on the immediate and long-term outcomes of children with Hirschsprung's disease. Pediatr Surg Int. 2006;22(2):179-181.

24. Mäkitie O, Heikkinen M, Kaitila I, Rintala R. Hirschsprung's disease in cartilage-hair hypoplasia has poor prognosis. J Pediatr Surg. 2002; 37(11):1585-1588.

25. Passarge E. The genetics of Hirschsprung's disease: Evidence for heterogeneous etiology and a study of sixty-three families. NEng/ J Med. 1967;276(3):138-143.

26. Bolk S, Pelet A, Hofstra RMW, et al. A human model for multigenic inheritance: Phenotypic expression in Hirschsprung disease requires both the RET gene and a new 9q31 locus. Proc Natl Acad Sci U S A. 2000;97(1):268-273.

27. Parisi MA, Kapur RP. Genetics of Hirschsprung disease. Curr Opin Pediatr. 2000;12(6):610-617.

28. Surana R, Quinn FM, Puri P. Evaluation of risk factors in the development of enterocolitis complicating Hirschsprung's disease. Pediatr Surg Int. 1994;9(4):234-236.

29. Lee CC, Lien R, Chian MC, et al. Clinical impacts of delayed diagnosis of Hirschsprung's disease in newborn infants. Pediatr Neonatol. 2012;53(2):133-137.
30. Sato A, Yamamoto M, Imamura K, Kashiki Y, Kunieda T, Sakata K. Pathophysiology of aganglionic colon and anorectum: An experimental study on aganglionosis produced by a new method in the rat. J Pediatr Surg. 1978;13(4):399-435.

31. Yoneda A, Shima H, Nemeth L, Oue T, Puri P. Selective chemical ablation of the enteric plexus in mice. Pediatr Surg Int. 2002;18(4):234-237.

32. Qin HH, Lei N, Mendoza J, Dunn JC. Benzalkonium chloride-treated anorectums mimicked endothelin-3-deficient aganglionic anorectums on manometry. J Pediatr Surg. 2010;45(12):2408-2411.

33. Webster W. Aganglionic megacolon in piebald-lethal mice. Arch Pathol. 1974;97(2):111-117.

34. Fujimoto T. Natural history and pathophysiology of enterocolitis in the piebald lethal mouse model of Hirschsprung's disease. J Pediatr Surg. 1988;23(3):237-242.

35. Fujimoto T, Reen DJ, Puri P. Inflammatory response in enterocolitis in the piebald lethal mouse model of Hirschsprung's disease. Pediatr Res. 1988;24(2):152-155.

36. Berg RD, Garlington AW. Translocation of certain indigenous bacteria from the gastrointestinal tract to the mesenteric lymph nodes and other organs in a gnotobiotic mouse model. Infect Immun. 1979;23(2): 403-411.

37. Awonuga AO, Belotte J, Abuanzeh S, Fletcher NM, Diamond MP, Saed GM. Advances in the pathogenesis of adhesion development: The role of oxidative stress. Reprod Sci. 2014;21(7):823-836.

38. Loftus TJ, Mohr AM, Moldawer LL. Dysregulated myelopoiesis and hematopoietic function following acute physiologic insult. Curr Opin Hematol. 2018;25(1):37-43.

39. Peters T, Peters JC. The biosynthesis of rat serum albumin. VI. Intracellular transport of albumin and rates of albumin and liver protein synthesis in vivo under various physiological conditions. J Biol Chem. 1972;247(12):3858-3863. 